\title{
Heterogeneity of failure of visual acuity in Graves' disease
}

\author{
Y. SACHDEV \\ M.D., D.C.H.
}

J. C. Chatterui

F.R.C.P.

\author{
R. C. Sharma \\ M.S.(Ophth.), D.O.
}

Endocrine Unit, Department of Medicine and Department of Ophthalmology, Army Hospital, New Delhi-110,010, India

\section{Summary}

The eye manifestations of Graves' disease are usually mild and self-limiting. Occasionally they follow a progressive course leading to visual loss and total blindness. The ocular manifestations bear no relationship to the metabolic state and may appear before, during or after onset of thyrotoxicosis. Characteristically they become evident at about the time of onset of hypermetabolism. Various factors responsible for the failure of visual acuity are discussed with case illustrations.

\section{Introduction}

The eye manifestations of Graves' disease are defined in 2 distinct categories: mild or noninfiltrative and severe or infiltrative (Werner, 1961). In the non-infiltrative variety, the common eye changes are prominent staring eyes, proptosis, wide palpebral fissure and lid retraction; while the infiltrative type is characterized by excessive lacrimation, grittiness, chemosis, decreased resilience of eye balls, ophthalmoplegias and fundal changes. Sudden or progressive visual failure due to corneal ulceration, corneal oedema and infiltration, papilloedema and optic atrophy may also be seen. Such cases are rare and usually the ophthalmic manifestations of Graves' disease are a self-limiting process (Brain, 1959).

\section{Clinical features}

For the purpose of this article, the clinical cases have been classified into 3 groups: where visual failure was due to - oedema and infiltration, $A$; involvement of the optic nerve, $B$; a combination of factors, C.

The clinical profile of 14 patients is given in Table 1.

Correspondence: Col. Y. Sachdev, Endocrine Unit and Radioimmunoassay Research Laboratories, Army Hospital, New Delhi-110,010, India.

\section{Discussion}

Exophthalmos goitre was first described by Guiseppe Flajani in 1802, but his description was considered too meagre and inaccurate to merit a serious consideration (Leading Article, 1968). In 1825 , the descriptions of 8 cases of exophthalmic goitre were found among the posthumous writings of Caleb Hillier Parry. Graves described it in 1835 while Basedow described its ophthalmic features in 1840 (Leading Article, 1968). Many terms (progressive exophthalmos, infiltrative ophthalmopathy, dysthyroid eye disease, thyrotoxic exophthalmos, malignant exophthalmos, exophthalmic ophthalmoplegia) have been proposed to describe the infiltrative type of ophthalmopathies associated with Graves' disease (Havard, 1972), but most of these do not signify the true nature of the underlying pathology (Taylor, 1960). The term 'congestive ophthalmopathy' is preferred as the lids and conjunctivae are invariably congested and oedema is a frequent feature. The American Thyroid Association (Werner, 1969) have adopted a classification where the 'mild' end of the spectrum is represented by class 1 and the 'severe' end by classes 2-6. Since eye changes may persist after the disease process has subsided, the terms 'active' and 'inactive' are added.

The clinical impression which persisted for over a century was that the ocular manifestations are of purely hyperthyroid origin and occur only with Graves' disease. Over the last 2 decades, however, clinical and experimental studies have shown that the progressive eye changes can appear in euthyroid and hypothyroid state; and may even begin before there is any evidence of thyroid dysfunction (Werner and Ingbar, 1971). This is supported by the authors' experience. Most of their patients were either euthyroid or under treatment for hyperthyroidism while others were frankly hypothyroid. The rate of control of the hyperthyroid state rather than 


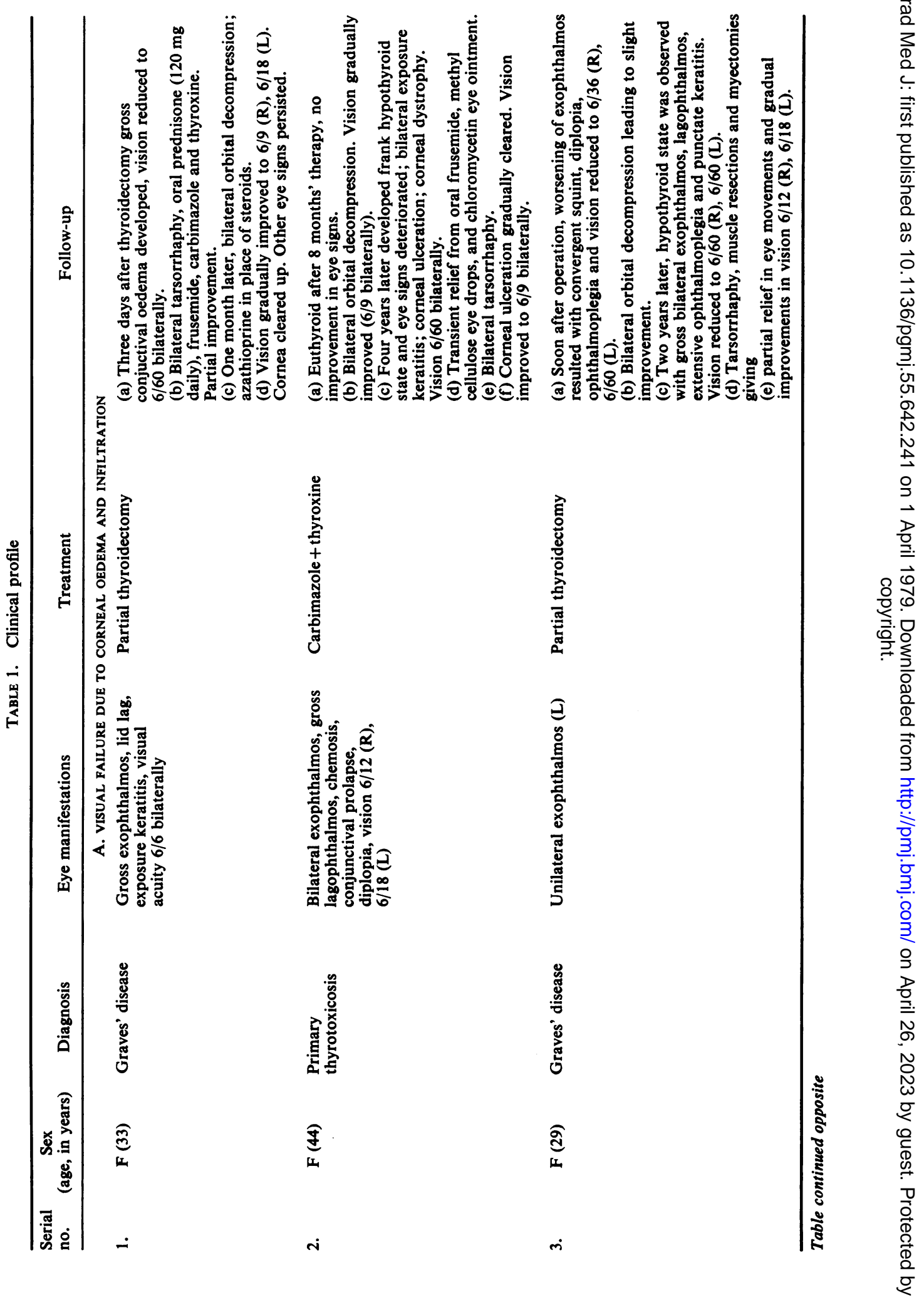


욤

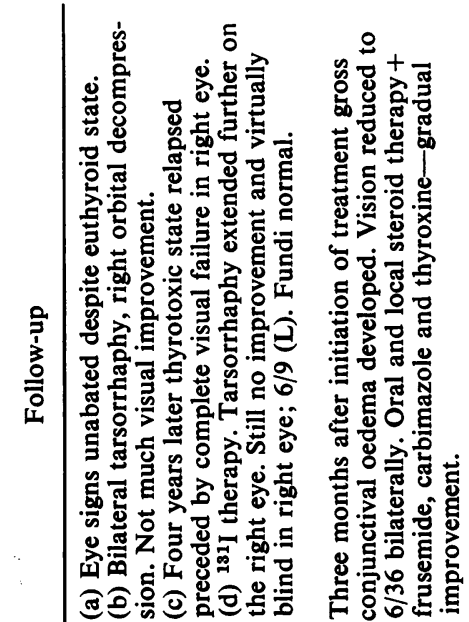

홍요

穿造造

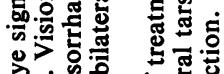

बें

iิ

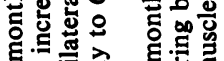

○.ด्ड

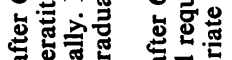

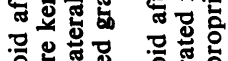

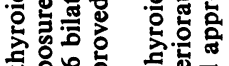

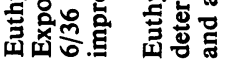

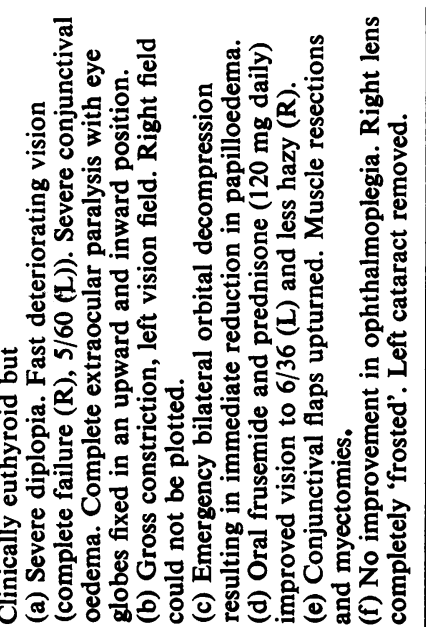

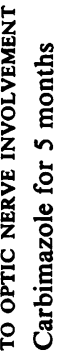

"山.

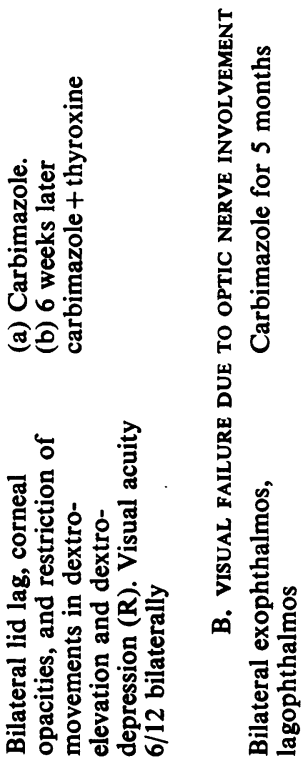

苛

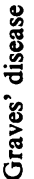

$\underset{\Sigma}{\Sigma}$

$\infty$

윽

ㄱ.

ชิ

$\stackrel{N}{ \pm}$

옥

을

응

훅잉

ֻे

옥

릉

흑

옥

ㅇํㄱ

음

을

N

N

웅

음

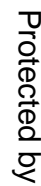



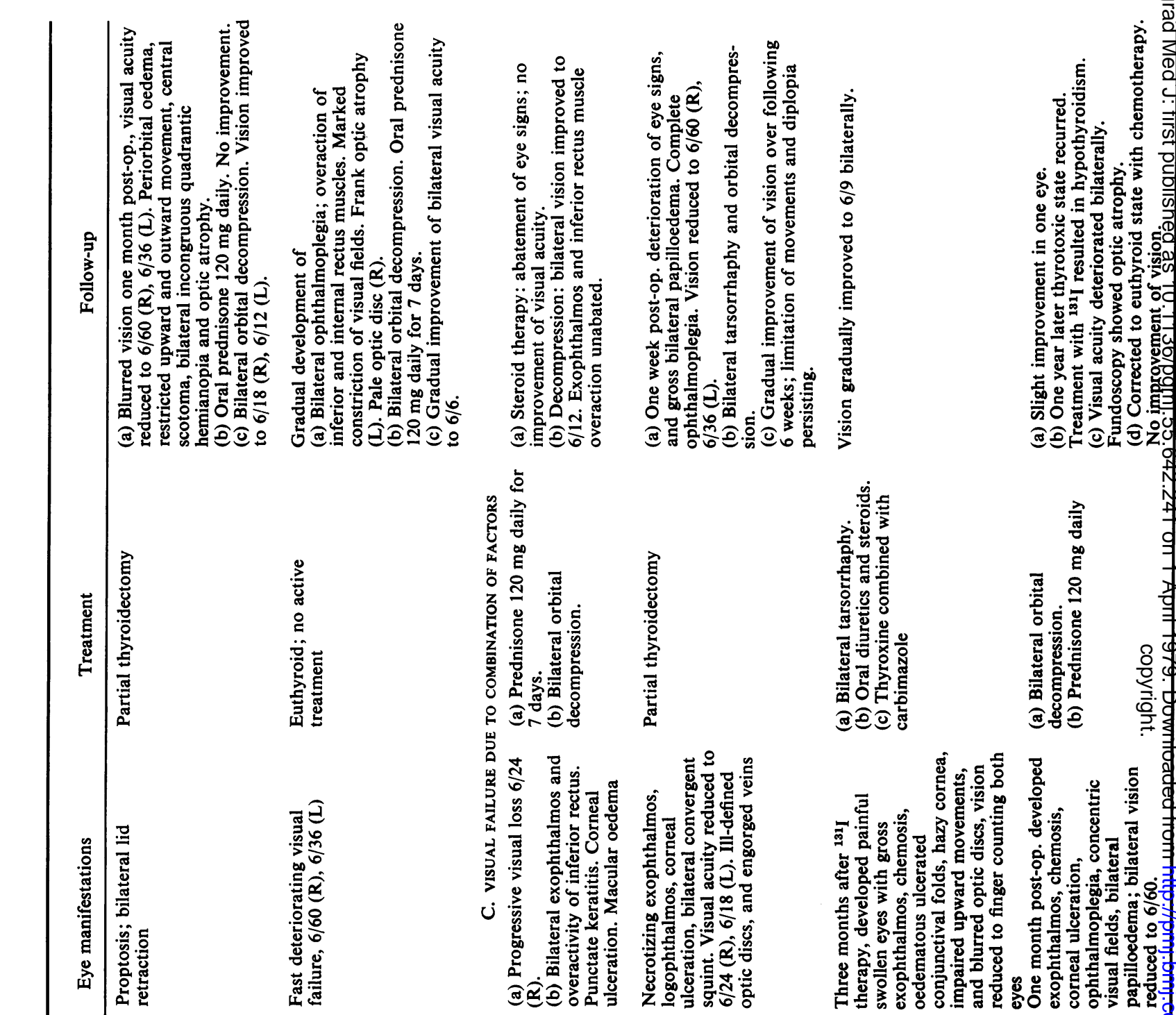
development of hypothyroidism may be the significant factor. Thus, rapid treatment tends to worsen the ocular manifestations (Aranow and Day, 1965). In some patients, the eye changes were aggravated by thyroid surgery although no definite time relationship was noticed. The incidence of progressive eye changes following subtotal thyroidectomy has been reported as 3 to $7 \%$ while following radioiodine therapy it is said to be approximately 5\% (Sloan, 1951; Hamilton et al., 1967). Zimmerman (1929) and Thomas and Woods (1936) have described many cases with progressive ocular changes which were initiated by partial thyroidectomy.

The course of exophthalmos and other eye changes is variable and unpredictable (Havard, 1972). In most of the patients it does not progress and behaves as a self-limiting process. What heralds an end is not known. Sometimes it may take years to appear and stay static for prolonged periods or progress haltingly. Hales and Rundle (1960) found progressive exophthalmos in only 24 out of 104 patients followed-up over a period of 15 years.

Visual failure was the result of corneal dystrophy in 7 of 14 patients with Graves' disease. Two patients had aggravation of ocular manifestations soon after partial thyroidectomy. In the other 5, although the euthyroid status was achieved easily with carbimazole, propranolol and thyroxine, the eye manifestations remained unabated and required surgical intervention in the form of bilateral decompression and tarsorrhaphy. The improvement in the eye signs was slow, incomplete and unrelated to the progress in the thyroid state. The optic nerve involvement was responsible for visual failure in 3 patients (group B). Papilloedema, optic atrophy, constricted visual fields and ophthalmoplegias of various types were the main features and required early (and at times urgent) orbital decompression, muscle resections, myectomies, oral diuretics and oral and local corticosteroids. There were 4 patients in group C. In this group, the manifestations of corneal dystrophy and involvement of the macular area, optic disc and extra-ocular muscles were present in different combinations. Surgery with judicious use of corticosteroids and diuretics improved the vision to a certain extent. The improvement in this group was slower and incomplete compared to that in the other 2 groups.

\section{Pathogenesis}

The pathogenesis of ocular changes is still an enigma. The biopsy and post-mortem specimens of orbital tissue of human and experimental animals have shown an increase in the ground substance of the connective tissue, hyaluronic acid, mast cells, fibroblasts and lymphocytes. The histopathological picture is consistent with pathological noninflammatory immune infiltration. Thyroid stimulating immunoglobulins (TSI) appear to be responsible. Thyrotrophic hormone (TSH), exophthalmosproducing substance (EPS), and long-acting thyroid stimulator (LATS) have also been incriminated in the aetiopathogenesis of ocular manifestations (Adams and Purves, 1957; Dobyns, Wright and Wilson, 1961; Kriss et al., 1967; McKenzie and McCullagh, 1968). Two recent studies have strengthened the impression that eye disorders are systemically related (Winand, 1968; Pisarev, Altschuler and Davison (1968). Depending upon the degree of exophthalmos and lagophthalmos, other complications such as exposure keratitis, corneal ulceration, severe hypopyon keratitis, and even panophthalmitis may result. The heavy and congested lids pressing on the cornea also contribute to corneal ulceration. Ophthalmoplegia is a troublesome complication and responds poorly to treatment. The anatomical position of the extra-ocular muscles is such that with their sheaths and orbital fascia they form a cone which is anchored to the orbit posteriorly and the base is formed by the eye. When the intra-orbital pressure is increased experimentally, both exophthalmos and immobility of the eye result (Poppen, 1953). The levator palpebrae superioris muscle is not stretched by the rise of intra-orbital tension and is rarely paralysed. This is attributed to the fact that its anterior insertion in the upper lid is mobile, not fixed. As the process advances, the affected muscles become fibrotic and short, leading to a fixed strabismus. The inferior rectus is the muscle which is most commonly involved. Bilateral shortening of this muscle accounts for the limitation in the upward movement commonly seen in clinical practice. With extensive muscle involvement the globe may be virtually immobile and the eyes are usually fixed in a downward squint. Very rarely the eye may be fixed in other positions. With increased intra-ocular pressure and overfilling of the orbit, the eyes become congested and the conjunctiva is glossy and waterlogged. Later it is thrown into folds along the free border of the lower lid. Subconjunctival haemorrhage and even glaucoma may result. The vision may fail from errors of refraction, corneal complications or optic nerve involvements. The optic nerve may be affected in the absence of severe proptosis. Increased intra-ocular pressure with resultant elevated pressure at the apex of the cone interferes with the blood supply of the optic nerve resulting in optic atrophy and irreversible visual loss. Poppen (1953) observed that increased tissue bulk confined in the region of the annulus of Zinn, where there was not enough fat to allow selfdecompression by fat absorption, resulted in 
pressure on the optic nerve, compromising its blood supply. Thus, in a patient who already has vascular disease, the vascular change could easily be precipitated. The visual prognosis in older patients and in those with arterial disease such as atherosclerosis and diabetes mellitus is, therefore, poor (Day and Carroll, 1962).

\section{Treatment}

Therapy is empirical and the main aim is to prevent corneal ulceration and optic nerve involvement. Simple lubrication drops such as $0.5 \%$ methyl cellulose, and opthalmic ointments may be used to help prevent drying. Eye pads with paraffin gauze dressings over the closed eyelids may be applied at night if there is incomplete closure of the eyelids. Antibiotics may be used locally for infection. Guanethidine drops (Crombie and Lawson, 1967) of $5 \%$ solution may be used to relieve eyelid retraction. Subconjunctival or retrobulbar injections of methylprednisolone had not given very encouraging results (Garber, 1966). Systemic steroid therapy has proved useful and sometimes in serious cases where the optic nerve is involved or corneal complications threaten, high doses of steroids given for a short period (prednisone 120 to $140 \mathrm{mg}$ daily for 7 days and gradually tapered off) may bring about the desired results (Werner, 1966). Glucocorticosteroids depress mucopolysaccharide synthesis and have a suppressive effect on the immune cells. Diuretics mobilize and promote the excretion of salt and water retained by the hydrophilic mucopolysaccharides and are useful. The role of immunosuppressive therapy is doubtful (Werner and Platman, 1965). Orbital decompression is undertaken when steroids are ineffective or contraindicated owing to systemic or local conditions. It is also advised when there is marked papilloedema, macular oedema or pressure on ciliary arteries manifesting as diminished visual acuity, field loss and marked increase in intra-ocular pressure. Classically, orbital decompression involves the removal of either the lateral wall or the roof of the orbit. More recently a transantral approach has been reported as giving good results (Ogura, Wessler and Avioli, 1971). By this method, the lateral wall of the ethmoidal sinuses and the roof of the maxillary sinus are removed. Surgical interference is not likely to benefit ocular movements as the muscle involvement is due to local infiltration of the muscle itself. An appropriate muscle resection and myectomy may help in a non-progressive phase. Radiotherapy to the pituitary, or hypophysectomy are no longer recommended. External irradiation of the orbital tissue is considered useful although its evaluation needs confirmation. Donaldson and Bagshaw (1972) have reported rapid and beneficial effects by highly collimated supravoltage radiation of the retro-orbital space. Ravin, Sisson and Knapp (1975) advise that orbital radiotherapy should be considered as an initial therapeutic approach to the optic neuropathy of Graves' disease. Treatment of a hypermetabolic state should be properly monitored and gradual progress in a hyperthyroid condition should be ensured to avoid hypothyroidism. In fact, the patient may even be allowed to be mildly hyperthyroid until eye complications terminate spontaneously or with treatment.

\section{Conclusion}

Corneal oedema and infiltration, ophthalmoplegia and optic nerve involvement are the usual factors responsible for the heterogeneity of visual failure in Graves' disease. Usually more than one factor is responsible. The integrity of the cornea must be of maintained with local antibiotics, large central tarsorrhaphy, orbital decompression (lateral or $N$

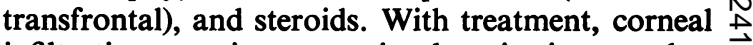
infiltration may improve, visual acuity increase but $\overrightarrow{\text { 을 }}$ proptosis, lid retraction and ophthalmoplegia usually persist. When the treatment is delayed, visual failure may lead to total blindness. A close and co-ordinated team approach by an endocrinologist and ophthalmologist is desirable for an effectivg treatment of ophthalmopathy.

\section{References}

ADAms, D.D. \& PuRves, H.D. (1957) The role of thyrotrophin in hyperthyroidism and exophthalmos. Metabolism, 6, 26.

ARANOW JR, H., \& DAY, R.M. (1965) Management of thyrotoxicosis in patients with ophthalmopathy. Antithyroid regimen determined primarily by ocular manifestations. Journal of Clinical Endocrinology, 25, 1.

BraIN, R. (1959) Pathogenesis and treatment of endocrine exophthalmos. Lancet, i, 109.

Crombie, A.L. \& LAwSON, A.A.H. (1967) Long term trial of local guanethidine in treatment of eye signs of thyroid dysfunctions and idiopathic lid retraction. British Medical Journal, 4, 592.

DAY, R.M. \& CARRoll, F.D. (1962) Optic nerve involvement associated with thyroid dysfunction. Archives of Ophthalmology, 67, 289.

Dobyns, B.M., Wright, A. \& Wilson, L. (1961). Assay of the exophthalmos producing substance in the serum of patients with progressive exophthalmos. Journal of $D$ Clinical Endocrinology, 21, 648.

Donaldson, S.S. \& BAGSHAw, M.S. (1972) Supravoltage orbital radiotherapy for Graves' ophthalmopathy. Forty- N eighth Meeting of the American Thyroid Association, p. 55.

GARBER, M.I. (1966) Methyl prednisolone in the treatment of exophthalmos. Lancet, i, 958.

HALES, I.B. \& RuNDLE, F.F. (1960) Ocular changes in Graves' disease. Quarterly Journal of Medicine, 29, 113.

Hamilton, R.D., MayberRy, W.E., MCConahey, W.M. \& Hanson, K.C. (1967) Ophthalmopathy of Graves' Disease: A comparison between patients treated surgically and patients treated with radio iodide. Proceedings. Mayo Clinic, 42, 812.

HAVARD, C.W.H. (1972) Endocrine exophthalmos. British Medical Journal, i, 360. 
Kriss, J.P., Pleshakov, V., Rosenblum, A.L., Holderness, M., Sharp, G. \& UTiger, R. (1967) Studies on the pathogenesis of the ophthalmopathy of Graves' disease. Journal of Clinical Endocrinology, 27, 582.

LEADING ARTICLE (1968) Management of endocrine ophthalmopathy. British Medical Journal, 3, 565.

McKenzie, J.M. \& McCullaGh, E.P. (1968) Observations against a causal relationship between long acting thyroid stimulator and ophthalmopathy in Graves' disease. Journal of Clinical Endocrinology, 28, 1177.

OgurA, J., Wessler, S. \& Avioli, V.L. (1971) Surgical approach to the ophthalmopathy of Graves' disease. Journal of the American Medical Association, 216, 1627.

Pisarev, M.A., Altschuler, N. \& Davison, T.A. (1968) Immune exophthalmos due to Harderian gland antigen. Endocrinology, 83, 903.

PoPPEN, J.L. (1953) Intractable exophthalmos in thyroid disease. Postgraduate Medicine, 14, 130.

Ravin, G.J., Sisson, C.J. \& KNAPP, T.W. (1975) Orbital radiation for ocular changes of Graves' disease. American Journal of Ophthalmology, 79, 285.

SlOAN, L.W. (1951) Surgical treatment of hyperthyroidism. New York Journal of Medicine, 51, 2897.
TAYlor, S. (1960) Eye signs in Graves' disease. Lancet, ii, 1187.

WERnER, S.C. (1961) The severe eye changes of Graves' disease. Journal of the American Medical Association, 177, 551.

WeRner, S.C. (1966) Prednisone in emergency treatment of malignant exophthalmos. Lancet, i, 1004.

WERNER, S.C. (1969) Classification of the eye changes of Graves' disease. Journal of Clinical Endocrinology, 29, 782.

Werner, S.C. \& Ingbar, S.H. (1971) The Thyroid. A Fundamental and Clinical Test. 3rd edn, p. 528. Harper and Row, London.

Werner, S.C. \& Platman, S.R. (1965) Remission of hyperthyroidism (Graves' disease) and altered pattern of serum-thyroxine binding induced by prednisone. Lancet ii, 751.

WINAND, R.J. (1968) Increased urinary excretion of acidic mucopolysaccharides in exophthalmos. Journal of Clinical Investigation, 47, 2563.

ZIMMERMAN, L.M. (1929) Exophthalmos following operation for the relief of hyperthyroidism. American Journal of Medical Science, Philadelphia, 178, 92. 